\title{
Global and local genetic diversity at two microsatellite loci in Plasmodium vivax parasites from Asia, Africa and South America
}

Mette L Schousboe ${ }^{1,2}$, Samir Ranjitkar ${ }^{1,2}$, Rupika S Rajakaruna ${ }^{3}$, Priyanie H Amerasinghe ${ }^{4}$, Flemming Konradsen ${ }^{1,2}$, Francisco Morales ${ }^{5}$, Rosalynn Ord ${ }^{6}$, Richard Pearce ${ }^{6}$, Toby Leslie $^{6}$, Mark Rowland ${ }^{6}$, Nahla Gadalla ${ }^{6}$, Ib C Bygbjerg ${ }^{1,2}$, Michael Alifrangis ${ }^{1,2}$ and Cally Roper ${ }^{6^{*}}$

\begin{abstract}
Background: Even though Plasmodium vivax has the widest worldwide distribution of the human malaria species and imposes a serious impact on global public health, the investigation of genetic diversity in this species has been limited in comparison to Plasmodium falciparum. Markers of genetic diversity are vital to the evaluation of drug and vaccine efficacy, tracking of $P$. vivax outbreaks, and assessing geographical differentiation between parasite populations.

Methods: The genetic diversity of eight $P$. vivax populations $(n=543)$ was investigated by using two microsatellites (MS), m1501 and m3502, chosen because of their seven and eight base-pair (bp) repeat lengths, respectively. These were compared with published data of the same loci from six other $P$. vivax populations.

Results: In total, 1,440 P. vivax samples from 14 countries on three continents were compared. There was highest heterozygosity within Asian populations, where expected heterozygosity $\left(H_{e}\right)$ was $0.92-0.98$, and alleles with a high repeat number were more common. Pairwise $F_{S T}$ revealed significant differentiation between most $P$. vivax populations, with the highest divergence found between Asian and South American populations, yet the majority of the diversity ( $89 \%)$ was found to exist within rather than between populations.

Conclusions: The MS markers used were informative in both global and local $P$. vivax population comparisons and their seven and eight bp repeat length facilitated population comparison using data from independent studies. A complex spatial pattern of MS polymorphisms among global $P$. vivax populations was observed which has potential utility in future epidemiological studies of the $P$. vivax parasite.
\end{abstract}

Keywords: Malaria, Plasmodium vivax, Genotyping, Microsatellites, Diversity, Heterozygosity

\section{Background}

Even though the malaria parasite Plasmodium vivax has the widest global distribution of the five human malaria species and imposes a serious impact on global public health, descriptions of global genetic diversity of this parasite are more limited than for Plasmodium falciparum. A recent study comparing the total genomic diversity among six $P$. vivax isolates drawn from Asia, Africa and South America with a comparable set of six $P$. falciparum

\footnotetext{
* Correspondence: Cally.Roper@lshtm.ac.uk

${ }^{6}$ Faculty of Infectious and Tropical Diseases, London School of Hygiene and Tropical Medicine, Keppel Street, London WC1 4HT, UK

Full list of author information is available at the end of the article
}

isolates reported twice as much single nucleotide polymorphism (SNP) diversity and a far deeper divergence among the $P$. vivax geographic strains [1]. There are accumulating reports of clinically severe disease and drugresistant $P$. vivax in some parts of the world [2-8], which reinforce the need for more detailed exploration of the global geography of $P$. vivax genetic diversity.

In the past, the most commonly used genetic markers for genotyping of $P$. vivax malaria parasites were antigencoding genes, such as the gene encoding the circumsporozoite protein, $P v c s p$, and the genes coding for the merozoite surface proteins Pvmsp-1 and Pvmsp-3 $\alpha$ [9-13]. Other antigen-coding genes such as Pvgam-1, Pvdbp, Pvama-1 
were used less frequently, possibly because of limited diversity of the individual loci [reviewed by [14]]. Comparison between populations using these markers has been complicated by differences in allele identification protocols used in different laboratories.

In recent years, microsatellites (MS) as short tandem repeats found throughout the $P$. vivax genome [15] have increasingly been used in studies of genetic diversity in P. vivax. As MS are generally non-coding, they are not subject to the same selective forces as antigen-encoding genes, and are thus more suitable for the analysis of $P$. vivax population structure $[15,16]$. Commonly, multiple MS loci found on different chromosomes are used in studies of population structure [6,17-20]. Repeat number polymorphism observed in a MS is the result of replication slippage that occurs during DNA replication, when the new strand mispairs with the template strand, and the degree of polymorphism is proportional to the underlying rate of mutation and the effective population size $[15,21]$. Slippage events become more common as the total number of repeats increase [19]. Hence, short repeat arrays tend to be less polymorphic than longer repeat arrays [1,22].

Most MS-based studies of genetic diversity and population structure of $P$. vivax parasites focus on one particular country or region, but three studies have compared population level diversity between countries or regions $[17,19,20]$, with sample sets between 214 to 425 samples. Two of the studies use a 13-14 tri- and tetra-nucleotide repeat MS $[19,20]$, while the third study analyses nine MS with repeat lengths of two to eight base-pair (bp) [17]. All the studies have revealed high levels of microsatellite diversity, with few private alleles being unique to populations or a specific geographical area.

The amalgamation of data from independent studies analysing genetic diversity at MS is hindered by use of different MS markers and further compounded by difficulties in the standardization of fragment size estimation on different sequencer machines. To simplify comparison between studies, a uniform set of MS loci is needed. Selection of MS with repeat lengths above four bp possibly improves the repeatability of allele classification among different laboratories as has been observed for P. falciparum MS studies [16].

In the present study the genetic diversity of samples from Sri Lanka, Nepal, Pakistan, and Afghanistan in South Asia, Venezuela and Ecuador in South America and Sudan and Sao Tome in Africa were analysed, using two MS m1501 (with seven bp repeat lengths) and m3502 (with eight bp repeat lengths) located on chromosome 1 and 3, respectively, initially described by Imwong et al. [17]. The data generated were compared with the published allele frequency data from Korea [6], India, Laos, Thailand and Colombia [17] and PNG [18]. The study evaluates the global and local genetic diversity at these two MS loci and assesses their differentiation among $P$. vivax populations worldwide.

\section{Methods \\ Sample collection}

The current study analysed $543 P$. vivax samples from Asia (Sri Lanka, Pakistan, Afghanistan, and Nepal), Africa (Sudan and São Tomé) and South America (Venezuela and Ecuador) and compared these data with published MS data from India, Thailand, Laos, Korea, PNG and Colombia described by others $[6,10,17,18]$. In total, 1,440 samples from 14 countries across three continents were compared, and the details of where and when these were collected are detailed in Table 1.

\section{Samples from Nepal}

Samples from Nepal were collected in two separate studies estimating the malaria burden in Nepal; 38 samples collected in 2009-2010 from Jhapa $(\mathrm{n}=33)$ and Banke $(\mathrm{n}=5)$ [23], and 17 samples in Kanchanpur $(\mathrm{n}=5)$ and Jhapa $(\mathrm{n}=12)$ in 2006 (Sean Hewitt, pers comm).

\section{Samples from Pakistan and Afghanistan}

The samples collected between 2004 and 2006 from closely linked sites in Pakistan $(\mathrm{n}=236)$ and Afghanistan ( $\mathrm{n}=93) 50 \mathrm{~km}$ apart were combined because of the similar demographic settings [24]. A second set of Pakistan samples $(n=139)$ was independently collected between 2004 and 2006 in Adizai, Baghicha, and Khagan villages, close to the North West Frontier Province Peshawar [25], while 60 additional samples were collected in the Ashaghroo refugee camp, Adizai, Pakistan (Kate Kolaczinski, pers comm).

\section{Samples from Ecuador}

In total, 17 samples were collected in the Sucumbíos Province in Ecuador in 2009 as a part of the Malaria transmission and natural resource management in the Ecuadorian Amazon project (Francisco Morales, pers comm).

\section{Samples from Venezuela}

The Venezuelan samples $(n=130)$ were from crosssectional malaria surveys conducted in ten communities along the Padamo River, Amazonas State in 1996 and $1997[12,26]$.

\section{Samples from Africa and São Tomé}

In Sudan, eight samples were collected in the village of Asar in Gedaref State during a community-based survey as a part of a $P$. falciparum artemether-lumefantrine efficacy trial during 2006 (Nahla Gadalla, pers comm). Four samples from São Tomé were collected in 2000 from 
Table 1 Heterozygosity $(\mathrm{He})$ at the two microsatellite loci $\mathrm{m} 1501$ and $\mathrm{m} 3502$ in every survey

\begin{tabular}{|c|c|c|c|c|c|c|c|c|c|c|c|}
\hline & Study site & $\mathbf{n}$ & Year & n (\%) & $\begin{array}{c}\mathrm{m} 1501 \\
\mathrm{He}\end{array}$ & MOI & n (\%) & $\begin{array}{c}\text { m3502 } \\
\mathrm{He}\end{array}$ & MOI & $\begin{array}{c}\mathrm{m} 1501+\mathrm{m} 3502 \\
n(\%)\end{array}$ & Ref \\
\hline \multirow[t]{8}{*}{ Asia } & Sri Lanka & 386 & $2002-2006$ & $352(91.2)$ & 0.85 & 1.276 & $357(92.5)$ & 0.74 & 1.073 & 338 (87.6) & *and [10] \\
\hline & Nepal & 55 & $2006,2009-2010$ & $53(96.4)$ & 0.94 & 1.189 & $49(89.1)$ & 0.80 & 1.347 & $47(85.5)$ & *and [23] \\
\hline & Pak/Afg & 329 & $2001,2003-2007$ & 315 (95.7) & 0.91 & 1.365 & $314(95.4)$ & 0.80 & 1.650 & $\begin{array}{c}309 \\
309(93.9)\end{array}$ & *and $[24,25]$ \\
\hline & Korea & 58 & 1996-2000, 2007 & $58(100)$ & 0.42 & - & $58(100)$ & 0.73 & - & $58(100)$ & {$[6]$} \\
\hline & India & 90 & 2003-2004 & 78 (86.7) & 0.90 & - & 79 (87.8) & 0.86 & - & - & {$[17]$} \\
\hline & Laos & 81 & $2001-2003$ & $81(100)$ & 0.83 & - & $74(91.4)$ & 0.90 & - & 74 (91.4) & {$[17]$} \\
\hline & Thailand & 92 & 1992-1998 & $91(98.9)$ & 0.89 & - & 91 (98.9) & 0.85 & - & - & [17] \\
\hline & PNG & 108 & $2004-2005$ & 107 (99.1) & 0.91 & 1.907 & $108(100)$ & 0.86 & 1.648 & $107(99.1)$ & [18] \\
\hline \multirow[t]{3}{*}{ South A. } & Columbia & 82 & 2001-2003 & $80(97.6)$ & 0.70 & - & $82(100)$ & 0.76 & - & 80 (97.6) & [17] \\
\hline & Venezuela & 130 & 1996-1997 & $113(86.9)$ & 0.73 & 1.416 & $98(75.4)$ & 0.57 & 1.235 & $97(74.6)$ & * \\
\hline & Ecuador & 17 & 2009 & $17(100)$ & 0.22 & 1.176 & 17 (100.0) & 0.76 & 1.176 & $17(100.0)$ & * \\
\hline \multirow[t]{3}{*}{ Africa } & Sudan & 8 & 2006 & $7(87.5)$ & 0.71 & 1.571 & $4(50.0)$ & 1.00 & 1.235 & $3(37.5)$ & * \\
\hline & São Tomé & 4 & 2000 & $4(100.0)$ & 0.83 & 1.250 & $4(100.0)$ & 0.83 & 2.250 & $4(100.0)$ & * \\
\hline & $\mathrm{n}=$ & 1440 & $1992-2010$ & $1356(94.2)$ & & 1.614 & 1335 (92.7) & & 1.490 & $1134 / 1258(90.1)$ & \\
\hline
\end{tabular}

PNG: Papua New Guinea. Pak/Afg: Pakistan/Afghanistan. South A: South America. The number (and percentage) of samples successfully amplified for each MS locus and for the two loci combined are shown in every survey. Multiplicty of infection (MOI) is calculated by averaging the number of alleles detected in the total number of PCR positive samples. Data not available are indicated by "-". Reference marked as * is this study.

Riboque $(n=3)$ and Porto Alegre $(n=1)$, kindly provided by Dr João Pinto.

\section{Samples from Sri Lanka}

Samples from Sri Lanka were collected 2002-2006 from multiple sites across Sri Lanka $(\mathrm{n}=386)$ [10].

\section{Published studies}

There are published data on allelic polymorphism at the same MS loci in $P$. vivax populations in Colombia $(\mathrm{n}=$ $82)$, India $(n=90)$, Laos $(n=81)$, and Thailand $(n=92)$ previously described by Imwong et al. [17]. Other studies have described polymorphism in samples from Korea $(\mathrm{n}=58)$ [6] and Papua New Guinea (PNG) $(\mathrm{n}=108)$ [18]. The samples from PNG were used only to compare expected heterozygosity values $\left(\mathrm{H}_{\mathrm{e}}\right)$ as the frequency of the individual alleles is not published [19].

\section{Amplification and fragment analysis of the Plasmodium vivax samples}

The $543 P$. vivax samples analysed were available either as blood spots on filter paper, as DNA already extracted from filter paper (using the Chelex-100 method [27] with modifications detailed in [28]) or as a blood smear on glass slides. The blood smear samples were a subset of the Nepalese samples and the method of DNA extraction is described in [23]. Sample size of the individual locations, year of collection, number of amplified samples, and percentage of polyclonal samples per locus are shown in Table 1.
The two MS, m1501 and m3502, were amplified by a semi-nested PCR and analysed on an ABI 3730XL genetic analyzer (Applied Biosystems, Foster City, CA, USA) using primers described by Imwong et al. [17], using a procedure described previously [10]. The length of the PCR fragment was determined by reference to the Genescan 500 Liz size standard (Applied Biosystems), using Genemapper vs. 4.1 (Applied Biosystems). Repetition of PCR was performed with $2 \mu \mathrm{l}$ DNA template in the primary PCR whenever a sample was negative at one of the loci. If a sample was PCR negative at both loci, the sample was excluded from further analysis. Limited volumes of DNA in some individual samples from Venezuela, São Tomé, Sudan, and Pakistan prevented repeat analysis of a subset of these samples.

Determining whether a sample was mono- or polyclonal was based on analysis of electropherograms obtained by Genemapper. Polyclonality of samples was determined when $>$ one peak was seen in the electropherogram. The existence of more than one peak indicates the presence of multiple genotypes or clones within an infection. Multiplicity of infection (MOI) among a group of samples was calculated by dividing the total number of clones detected in all PCR-positive samples by the total number of PCRpositive samples.

For the estimation of allele frequencies one allele was counted per sample. This was to avoid oversampling of rare alleles. In the case of mixed infections, the 'major' allele was counted. The relative size of the peaks was used to establish major versus minor alleles; if one allele 
peak was twice the height of the other allele(s) then a major allele was assigned. If peaks were of equivalent size or minor peak greater than $50 \%$ of the major, the allele was chosen by computer randomization.

PCR fragment-length measurements were calibrated against known repeat number by sequencing a subset of 11 Pakistan samples on an ABI Prism 377 (Perkin-Elmer) using ExoSAP-IT PCR Clean-up Kit (GE Healthcare) and the Big Dye terminator reaction mix (Perkin-Elmer), and using the $\mathrm{m} 1501$ and $\mathrm{m} 3502$ primers. After sequencing, the individual haplotypes were aligned with the published sequence of the Sal-1 strain (GenBank accession number: AAKM01000015) and analysed by use of the DNASTARLasergene software. There was good concordance between estimations of repeat number based on the PCR fragment size and that confirmed by direct sequencing.

\section{Measures of diversity and population differentiation}

The analysis of allelic diversity measured in the $P$. vivax study populations used four tests: 1 ) expected heterozygosity $\left(\mathrm{H}_{\mathrm{e}}\right)$, which can be defined as the chance of drawing two different alleles from a population, ranging between $0-1$. It was calculated as $H_{e}=[n /(n-1)]\left[1-\sum \mathrm{pi}^{2}\right]$, where $n$ is the number of samples and pi is the frequency of the $\mathrm{i}^{\text {th }}$ allele. The estimation of $\mathrm{H}_{\mathrm{e}}$ for each of the two loci in $P$. vivax in PNG was taken from the original publication [18]; 2) computation of $F_{\mathrm{ST}}$ pairwise population genetic distances; 3 ) analysis of molecular variance (AMOVA) attributes the proportion of the total genetic variance which is found within populations (countries), between populations within groups (continents), and among groups; and, 4 ) isolation by distance (IBD) was used to test for any correlation between $F_{\mathrm{ST}}$ estimates and geographic distances using Mantel's test [29]. The calculations were performed by plotting pairwise $F_{\mathrm{ST}} /\left(1-F_{\mathrm{ST}}\right)$ against the natural logarithm of the geographical distance (in $\mathrm{km}$ ). Evaluation of IBD was performed by showing $R$ squared $\left(R^{2}\right)$, indicating the percentage of the variance explained by the model, the correlation coefficient $r$ as a measure of the degree of linear relationship between two variables and the P-value. The calculations in all tests were performed by including both mono- and polyclonal samples.

The software used for the calculations of $\mathrm{H}_{\mathrm{e}}$ was Excel add-in MS Toolkit software, whereas the program FSTAT was used to perform the pairwise $F_{\mathrm{ST}}$ estimates and IBD calculations with 10,000 permutations [29]. Lastly, linkage equilibrium (LD) and AMOVA calculations were performed in Arlequin software version 3.11 [30], and significance of the AMOVA results was assessed by a randomization test with 10,000 permutations.

\section{Isolation by distance (IBD)}

To enable calculations of IBD, geographical distances in $\mathrm{km}$ were determined by use of Google Earth [31]. The samples from India, Korea, Laos, Thailand, Ecuador, and Sudan were originally collected at one site in each country, while the remaining samples originated from two to ten sites per country. To simplify the IBD calculations, one site was chosen per country. In Sri Lanka, most samples were collected from the district of Anuradhapura, hence the site of Anuradhapura General Hospital was chosen. The majority from the Pakistan/Afghanistan sample collection was from the Peshawar district, hence this site was chosen. In Colombia, five sites were included in the study, with ten to 20 samples collected per site [17]. The Tumaco municipality is located approximately in the middle of the five districts, and so this was chosen to represent the Colombian samples. The Gedaref State in Sudan, Riboque in São Tomé, the Jhapa district in Nepal, the approximate middle of the DMZ between North and South Korea and the centre part of Rio Padamo (Venezuela) were chosen to represent these countries.

\section{Results}

In total, 543 samples from Sri Lanka, Nepal, Pakistan and Afghanistan, Venezuela, Ecuador, Sudan and Sao Tome were analysed. The number and percentage of samples successfully amplified at each locus are shown in Table 1. The rate of success was similar for m1501 (94.2\%) and m3502 (92.7\%) (Table 1). Additional MS data on m1501 and m3502 obtained from published studies are also included in Table 1 and the $\mathrm{H}_{\mathrm{e}}$ at each locus in every site is compared. A high $\mathrm{H}_{\mathrm{e}}$ was observed at both loci in all the sites, with variations among sites being broadly consistent across the two loci. The South American sites had lower diversity $\left(\mathrm{H}_{\mathrm{e}}\right.$ range: $\left.\mathrm{m} 1501=0.22-0.73, \mathrm{~m} 3502=0.57-0.76\right)$ than Asian $\left(\mathrm{H}_{\mathrm{e}}\right.$ range: $\mathrm{m} 1501=0.42-0.94, \mathrm{~m} 3502=0.73$ $0.90)$ and African sites $\left(H_{e}\right.$ range: $m 1501=0.71-0.83$, $\mathrm{m} 3502=0.83-1.00)$.

\section{Standardizing repeat number at microsatellites $\mathrm{m} 1501$ and $\mathrm{m} 3502$}

For the analysis of genetic differentiation between populations, independently derived data from published studies of P. vivax in Thailand, Laos, India and Colombia published by Imwong et al. [17] and from Korea published by Honma et al. [6] were incorporated in the analysis. Allele nomenclature prevented amalgamation of data from a study in PNG [18] in the meta-analysis. To combine these data with the data obtained from the present study, fragment sizes were grouped according to the estimated number of repeats under a common allele name. Additional files 1 and 2 show how alleles common to different studies were matched and how the dataset was unified.

A few m1501 alleles from Sri Lanka and Pakistan samples and m3502 alleles in samples from Laos and Sri Lanka showed irregular spacing caused by a fragment size shift of a few nucleotides, presumably caused by insertion/ 
deletion within the PCR amplified fragment. These irregular alleles were classified under the same repeat number as those of similar size elsewhere but are distinguished in the Tables by appending ' $a$ ' and ' $b$ ' to the repeat number. In the allele frequency and Fst calculations subgroups a and $\mathrm{b}$ were combined [see Additional files 1 and 2].

\section{Polymorphism at microsatellites $\mathrm{m} 1501$ and $\mathrm{m} 3502$}

The m1501 marker discriminated 29 alleles in the global dataset while $\mathrm{m} 3502$ discriminated 18 alleles. The number of alleles differed remarkably between populations. Asian $P$. vivax populations were highly polymorphic at both loci, with the exception of the Korean $P$. vivax population. This contrasted with the South American P. vivax populations, which were less diverse. Diversity at $\mathrm{m} 1501$ was highest in Pakistan where 27 alleles were detected among 315 samples, while only two were seen in Ecuador $(n=17)$ and three in Korea $(n=58)$ [see Additional file 1]. For the m3502 marker, again, the highest diversity was found in
Pakistan, while the lowest diversity was observed in the African and Korean samples where only three alleles and four alleles per site were detected, respectively [see Additional file 2]. For both markers, the most common alleles had a repeat size ranging from three to six repeats. This was true among all study populations except Korea, where a 13-repeat m3502 allele, named 216, comprised $39.7 \%$ of the 58 samples.

Alleles with long fragment lengths, above 12 repeats for $\mathrm{m} 1501$ and 10 repeats for $\mathrm{m} 3502$, were detected more commonly in Asian than elsewhere (Figure 1A and B). In general, the maximum number of repeats seen in South America and Africa was 17 repeats for $\mathrm{m} 1501$ and 11 repeats for m3502 repeats while in Asia, repeat lengths of up to 31 for $\mathrm{m} 1501$ and 19 for m3502 were common. An exception to this trend was two samples from Venezuela where long-fragment m1501 alleles of 31 repeats were observed, while a 21-repeat $\mathrm{m} 3502$ allele was detected in Sudan.

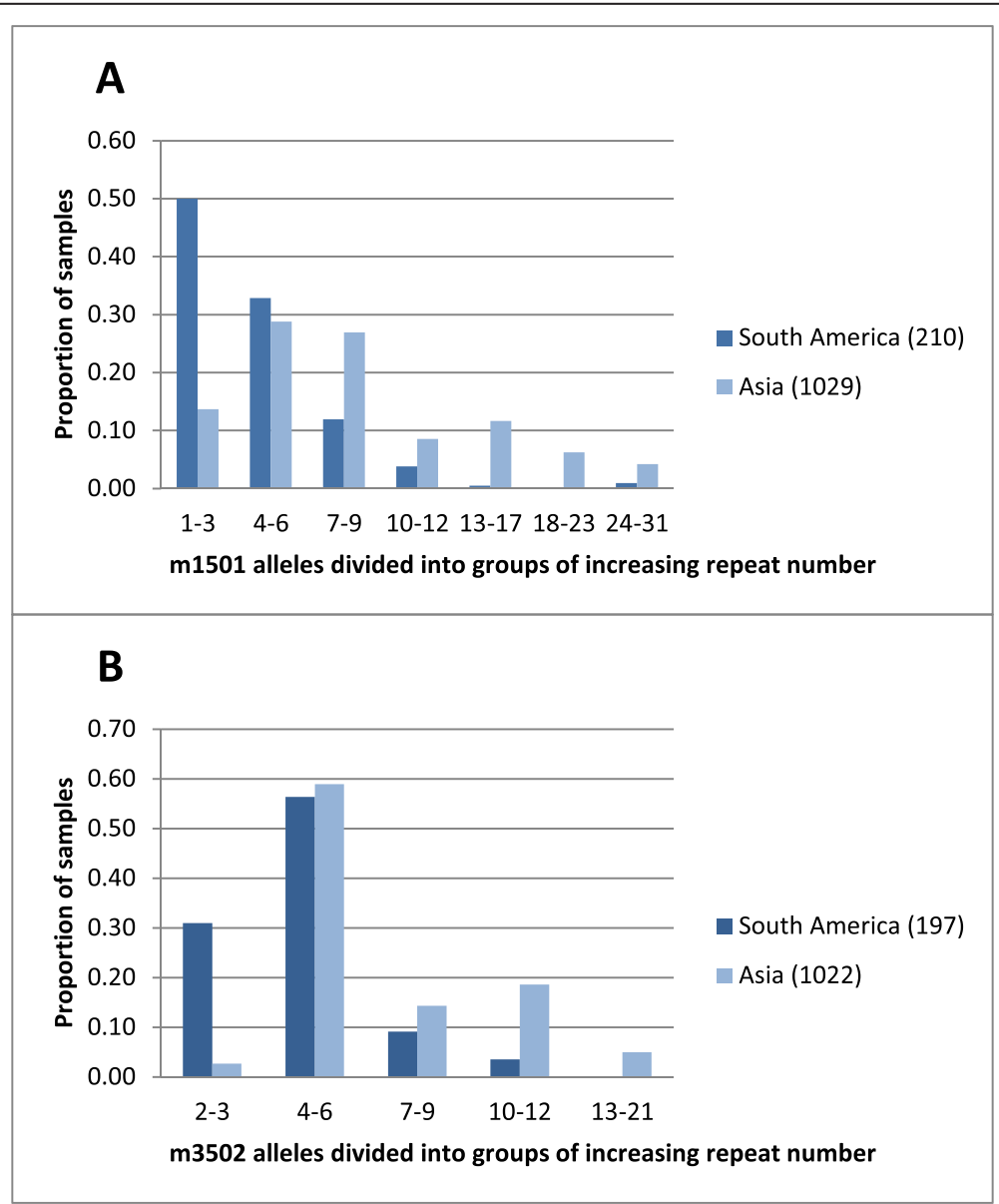

Figure 1 Allelic diversity at the MS $\mathrm{m} 1501$ (A) and $\mathrm{m} 3502$ (B) in P. vivax samples from South America (Columbia, Venezuela and Ecuador) and Asia (India, Laos, Thailand, Korea, Nepal, Pakistan and Sri Lanka). The MS alleles are divided into groups of increasing fragment size according to the number of repeats. In brackets are mentioned the number of samples included from each continent. 


\section{Pairwise differentiation between Plasmodium vivax populations}

Plasmodium vivax populations in South America (Venezuela, Colombia and Ecuador) showed evidence of population structure. $F_{\mathrm{ST}}$ using the m1501 marker revealed a significant difference between Colombia and the two other South American countries $\left(F_{\mathrm{ST}}=0.05-0.21\right)$, while $F_{\mathrm{ST}}$ using the m3502 marker, found significant differences between Venezuela and the two other South American countries $\left(F_{\mathrm{ST}}=0.26-0.27\right) \quad$ [see Additional file 3]. Generally, $P$. vivax populations from these three countries were significantly different from all the Asian countries, with few exceptions. Low, but significant pairwise $F_{\mathrm{ST}}$ estimates were found among the Asian countries; 0.02-0.31 (m1501) and 0.02-0.14 (m3502). The analysis of IBD was done by using pairwise genetic and geographic distances. Evidence for IBD was found for both loci by Mantel's test; $\mathrm{m} 1501:\left(\mathrm{R}^{2}=6.35, \mathrm{r}=0.0789, \mathrm{P}=0.04\right)$, and m3502: $\left(\mathrm{R}^{2}=7.99, \mathrm{r}=0.0285, \mathrm{P}=0.02\right)$.

$F_{\mathrm{ST}}$ estimates using the two loci combined were slightly higher and pairwise estimates between $P$. vivax populations from Korea, Nepal, Pakistan, Sri Lanka, Venezuela, Ecuador, Sudan, and São Tomé, revealed $F_{\mathrm{ST}}$ between 0.01-0.40 [see Additional file 4]. The highest difference was found between the $P$. vivax populations in Ecuador and Sudan and the lowest between $P$. vivax populations from Nepal and Sri Lanka. The two-locus haplotypes were often not specific to a single sample set from one country, but present in more than one $P$. vivax population as for instance the '107-142' haplotype seen in Nepal, Pakistan and Sri Lanka [see Additional file 5].

\section{The allelic diversity within and between Plasmodium vivax} populations

The allelic diversity within the different $P$. vivax populations was estimated by $\mathrm{H}_{\mathrm{e}}$. The average $\mathrm{H}_{\mathrm{e}}$ per locus was $0.90(\mathrm{~m} 1501)$ and $0.84(\mathrm{~m} 3502)$. The $\mathrm{H}_{\mathrm{e}}$ varied considerably from site to site, but was generally lowest in the three South American countries $\left(\mathrm{H}_{\mathrm{e}}=0.22-0.76\right)$ and highest in the Asian populations $\left(\mathrm{H}_{\mathrm{e}}=0.80-0.94\right)$ with the exception of Korea, which possessed very low $\mathrm{H}_{\mathrm{e}}$ values of 0.42 (m1501) and 0.73 (m3502) (Table 1 and Additional file 6). The AMOVA analysis found the bulk ( 89\%) of genetic variance occurs within the $14 P$. vivax populations [see Additional file 7], $\sim 9 \%$ of the variance occurs between populations within continents, and only about $4 \%$ of the variance occurs between the continents [see Additional file 7].

Comparison of the three continents (South America, Asia and Africa) showed that the South American P. vivax population was distinct from the two other continents, with low but significant $F_{\mathrm{ST}}$ estimates $(\mathrm{m} 1501=0.09-0.18$, $\mathrm{m} 3502=0.08-0.11$ ) (Table 2). This may be attributable to the lower diversity found in South America where the
Table 2 Genetic differentiation between $P$. vivax populations measured by pairwise $\boldsymbol{F}_{\mathrm{ST}}$

\begin{tabular}{lccc}
\hline $\mathbf{m} 1501$ & Africa & South America & Asia \\
\hline Africa (11) & & $* *$ & NS \\
South America (210) & 0.1824 & & $* *$ \\
Asia (1028) & 0.0446 & 0.0869 & \\
\hline $\mathbf{m 3 5 0 2}$ & Africa & South America & Asia \\
\hline Africa (8) & & $* *$ & NS \\
South America (197) & 0.1050 & & $* *$ \\
Asia (1022) & 0.0049 & 0.0752 & \\
\hline
\end{tabular}

The pairwise significance after standard Bonferroni corrections are listed as: "**" significance at the $1 \%$ nominal level while "NS" stands for non-significant.

m1501 marker possessed the lowest $H_{e}$ values $(n=210$, $\left.\mathrm{H}_{\mathrm{e}}=0.71\right)$, thereafter Africa $\left(\mathrm{n}=11, \mathrm{H}_{\mathrm{e}}=0.84\right)$ whereas Asia had the most diverse $P$. vivax populations in this study $\left(n=1,028, H_{e}=0.92\right)$. The same ranking was seen using the m3502 marker: South America $\left(n=197, \mathrm{H}_{\mathrm{e}}=\right.$ $0.76)$, Africa $\left(n=8, H_{e}=0.86\right)$ and Asia $\left(n=1,022, H_{e}=\right.$ $0.84)$.

\section{Discussion}

The main objective of the current study was to evaluate the global genetic diversity of $P$. vivax populations by examining the two MS, m1501 and m3502 in P. vivax samples collected from Ecuador, Venezuela, Sri Lanka, Afghanistan, Pakistan, Nepal, São Tomé, and Sudan (n = 543). Although some of these counties were represented by a large $P$. vivax sample set, the limited available sample set from Ecuador $(\mathrm{n}=17)$, São Tomé $(\mathrm{n}=4)$ and Sudan $(\mathrm{n}=$ 8) means caution should be applied to the interpretation of the results regarding these sites. Furthermore, there is a significant time interval between the collection of the Venezuelan samples in 1996-1997 and the other South American samples some years later. Where possible, the resulting data were compared with published data from studies of the same markers in Colombia, India, Laos, Thailand, PNG, and Korea $(\mathrm{n}=897)$.

The geographical genetic diversity of the $P$. vivax populations was shown to be highly diverse, with the majority of the diversity found to be present within the populations ( 89\%). In total, $29 \mathrm{~m} 1501$ and $18 \mathrm{~m} 3502$ alleles were detected with average $\mathrm{H}_{\mathrm{e}}$ estimates per allele of 0.90 (m1501) and 0.84 (m3502), lowest in the South American samples and highest in Asia. The $\mathrm{H}_{\mathrm{e}}$ values found in this study by use of only two MS $\left(\mathrm{H}_{\mathrm{e}}=0.78\right.$ 0.98) were high compared to $\mathrm{H}_{\mathrm{e}}$ estimates reported by other studies $\left(\mathrm{H}_{\mathrm{e}}=0.48-0.87\right)$, which have analysed samples from the same three continents including nine to 14 MS $[6,17,20,32]$, and the $\mathrm{H}_{\mathrm{e}}$ estimates of 0.26 to 0.91 reviewed by others [14].

South American and Asian populations were found to be significantly different from each other by pairwise 
$F_{\mathrm{ST}}$, whereas the African population generally could not be distinguished statistically from most other populations, most probably this was due to the limited sample size. The $F_{\mathrm{ST}}$ estimates ranged from 0.02-0.63 (m1501) and 0.03-0.28 (m3502) per site, and a Mantel test correlating pairwise genetic and geographic distances between populations showed evidence for IBD.

The high frequency of alleles with low repeat number (one to six repeats) was common for all the $P$. vivax populations, and the rarer long-repeat fragments were mainly restricted to Asia. Only a limited number of samples from Africa were analysed, but these suggest Africa is intermediate between South American and Asian populations. The long repeat-alleles appearing in the Asian $P$. vivax populations might relate to factors, such as higher $P$. vivax endemicity in Asian countries.

Malaria endemicity is expected to have a significant influence on genetic diversity and levels of inbreeding/outbreeding. Among the populations sampled in the present study, Asian populations were the most diverse, although a range of $\mathrm{H}_{\mathrm{e}}$ estimates and MOI values were measured among the individual populations sampled. The maximum MOI for an individual sample was six clones and this was found in Pakistan. The mean MOI in Pakistan was 1.55 and rates of heterozygosity were also high. The complexity of the Pakistan/Afghanistan population is striking, since these areas are not generally considered highly endemic. Similar rates of heterozygosity were reported in PNG [33] and the equivalent mean MOI value there was 1.82 . It is generally accepted that high polyclonality increases the probability for heterogametic genetic recombination during the sexual cycle occurring in the mosquitoes, resulting in sporozoites with novel genotypes [34]. This may also promote heterozygosity at MS loci through a process of strand slippage during recombination. The mutation rate of MS in the $P$. vivax genome is unknown, but the high rates of complexity observed here are fully consistent with that reported from previous studies of $P$. vivax $[10,32,35]$.

The above model of polyclonality and diversity is developed based on extensive and cumulative $P$. falciparum research, and is not the complete explanation of the mechanisms that contribute to $P$. vivax diversity. Genome wide analyses of MS diversity have shown $P$. vivax diversity is significantly greater than P. falciparum [1], but also that genome-wide SNP diversity is greater among $P$. vivax than $P$. falciparum [1]. The same study showed a longer time to most recent common ancestor among $P$. vivax isolates suggesting $P$. vivax diversity is more ancient. Although just six $P$. vivax isolates were compared, similar geographic trends were observed. South American isolates Brazil 1, Salvador 1 and Peru shared a more recent common ancestor with each other than with isolates from Asia (India, Korea) and Africa (Mauritania), which had deep branch lengths and did not cluster. The data presented in this study also showed high level of diversity and differentiation between the South American and Asian populations and the findings indicate that further investigation of genome-wide diversity among $P$. vivax populations from Central and Southeast Asia may reveal even greater levels of genetic diversity.

A ' $P$. falciparum-model' may not entirely predict and explain genetic diversity of $P$. vivax populations. Important biological differences between $P$. vivax and $P$. falciparum may also be at play. In $P$. vivax, gametocytogenesis occurs earlier in clinical episodes, and reticulocytes are essential to both invasion and relapse [36]. Significant linkage disequilibrium has been observed in the included sample set from Sri Lanka [10], the Colombian samples [17], the Korean samples [6], and by others analysing samples from Sri Lanka [19] and Brazil [35] and these findings suggest that clonal expansions of specific genotypes may be epidemiologically significant and further studies are needed to elucidate the local dynamics of $P$. vivax epidemics.

\section{Conclusions}

The data presented in this study show the utility of the MS m1501 and m3502 in studies of $P$. vivax population structure, irrespective of geographical origin, although more markers might be needed in hypo-endemic areas if distinguishing between individual parasites is a priority. Ultimately, whole- genome analyses will provide detailed estimation of the total genetic diversity -the results presented in this study suggests that the greatest diversity will be in Southeast Asia.

\section{Ethical statement}

Clearance for analysis of Plasmodium genes were approved by London School of Hygiene Tropical Medicine and Hygiene Ethics Board, locally by Bioethics Committee, Pakistan Medical Research Council and Directorate of Public Health, Jalalabad, Nangahar, Comitee de Bioetica Universidad San Francisco de Quito and the Nepal Health Research Council.

\section{Additional files}

Additional file 1: Allelic diversity at the m1501 locus of $P$. vivax samples obtained from various malaria endemic countries.

Additional file 2: Allelic diversity at the m3502 locus of $P$. vivax samples obtained from various endemic countries.

Additional file 3: Population differentiation at m1501 and m3502 allelic estimated by pairwise fixation index, $\mathrm{F}_{\mathrm{ST}}$.

Additional file 4: Population differentiation estimated by pairwise fixation index, $F_{\mathrm{ST}}$ using the combined MS data from m1501 and m3502.

Additional file 5: Allelic diversity for the combined MS genotype "m1501-3502" haplotypes per study site and in total. 


\section{Additional file 6: Expected Heterozygosity (He) two $P$. vivax} microsatellites, m1501 and 3502 in each site.

Additional file 7: Global analysis of molecular variance (AMOVA) among $P$. vivax populations and continents (groups).

\section{Competing interests}

The authors have declared that they have no competing interests.

\section{Authors' contributions}

$M L S$, with CR, ICB and MA designed the study. MLS carried out the molecular genetic studies, and with RP, RO, MA and CR participated in the sequence alignment and further analyses as well as statistical analysis. MLS drafted the manuscript and SR, RSR, PHA, FK, FM, RO, RP, TL, MR, NG, ICB, MA and CR commented the first draft and further revisions. All authors read and approved the final manuscript.

\section{Acknowledgements}

The Danish Agency for Science, Technology and Innovation are thanked for financial support to fulfil this study. Furthermore, Dr. Sean Hewitt, Dr. Kate Kolaczinski, Dr. Joao Pinto, Dr. Adriana Tami and Francisco Morales are gratefully acknowledged for supplying the P. vivax samples from Nepal, Pakistan, Sudan, São Tomé, Venezuela, and Ecuador. Dr. Line Vej Ugelvig, together with technicians Ruth Bruus Jakobsen and Lisbeth Knudsen (University of Copenhagen) are thanked for support with microsatellite analysis and statistical quidance. Furthermore, technician Ulla Abildtrup is thanked for her excellent support in the laboratory.

\section{Author details}

${ }^{1}$ Centre for Medical Parasitology, Institute of International Health, Immunology, and Microbiology, University of Copenhagen, Copenhagen, Denmark. ${ }^{2}$ Department of Infectious Diseases, Copenhagen University Hospital, Copenhagen, Denmark. ${ }^{3}$ Department of Zoology, University of Peradeniya, Peradeniya 20400, Sri Lanka. International Water Management Institute, Hyderabad, Andhra Pradesh, India. ${ }^{5}$ Robles Y Pampite, Cymbaya, Universidad San Francisco de Quito, San Francisco de Quito, Ecuador. ${ }^{6}$ Faculty of Infectious and Tropical Diseases, London School of Hygiene and Tropical Medicine, Keppel Street, London WC1 4HT, UK

Received: 9 May 2014 Accepted: 28 August 2014

Published: 2 October 2014

\section{References}

1. Neafsey DE, Galinsky K, Jiang RH, Young L, Sykes SM, Saif S, Gujja S, Goldberg JM, Young S, Zeng Q, Chapman SB, Dash AP, Anvikar AR, Sutton PL, Birren BW, Escalante AA, Barnwell JW, Carlton JM: The malaria parasite Plasmodium vivax exhibits greater genetic diversity than Plasmodium falciparum. Nat Genet 2012, 44:1046-1050.

2. Beg MA, Khan R, Baig SM, Gulzar Z, Hussain R, Smego RA Jr: Cerebral involvement in benign tertian malaria. Am J Trop Med Hyg 2002, 67:230-232.

3. Genton B, D'Acremont V, Rare L, Baea K, Reeder JC, Alpers MP, Müller I: Plasmodium vivax and mixed infections are associated with severe malaria in children: a prospective cohort study from Papua New Guinea. PLoS Med 2008, 5:e127.

4. Singh H, Parakh A, Basu S, Rath B: Plasmodium vivax malaria: is it actually benign? J Infect Public Health 2011, 4:91-95.

5. Tjitra E, Anstey NM, Sugiarto P, Warikar N, Kenangalem E, Karyana M Lampah DA, Price RN: Multidrug-resistant Plasmodium vivax associated with severe and fatal malaria: a prospective study in Papua, Indonesia. PLoS Med 2008, 5:e128.

6. Honma H, Kim JY, Palacpac NM, Mita T, Lee W, Horii T, Tanabe K: Recent increase of genetic diversity in Plasmodium vivax population in the Republic of Korea. Malar J 2011, 10:257.

7. Price RN, Douglas NM, Anstey NM: New developments in Plasmodium vivax malaria: severe disease and the rise of chloroquine resistance. Curr Opin Infect Dis 2009, 22:430-435.

8. Imwong M, Pukrittayakamee S, Renia L, Letourneur F, Charlieu JP, Leartsakulpanich U, Looareesuwan S, White NJ, Snounou G: Novel point mutations in the dihydrofolate reductase gene of Plasmodium vivax: evidence for sequential selection by drug pressure. Antimicrob Agents Chemother 2003, 47:1514-1521.

9. Cui L, Mascorro CN, Fan Q, Rzomp KA, Khuntirat B, Zhou G, Chen H, Yan G, Sattabongkot J: Genetic diversity and multiple infections of Plasmodium vivax malaria in Western Thailand. Am J Trop Med Hyg 2003, 68:613-619.

10. Schousboe ML, Rajakaruna RS, Amerasinghe PH, Konradsen F, Ord R, Pearce R, Bygbjerg IC, Roper C, Alifrangis M: Analysis of polymorphisms in the merozoite surface protein-3alpha gene and two microsatellite loci in $\mathrm{Sr}$ Lankan Plasmodium vivax: evidence of population substructure in Sri Lanka. Am J Trop Med Hyg 2011, 85:994-1001.

11. Kim JR, Imwong M, Nandy A, Chotivanich K, Nontprasert A, Tonomsing $N$, Maji A, Addy M, Day NP, White NJ, Pukrittayakamee S: Genetic diversity of Plasmodium vivax in Kolkata, India. Malar J 2006, 5:71.

12. Ord R, Polley S, Tami A, Sutherland CJ: High sequence diversity and evidence of balancing selection in the Pvmsp3alpha gene of Plasmodium vivax in the Venezuelan Amazon. Mol Biochem Parasitol 2005, 144:86-93.

13. Imwong $\mathrm{M}$, Pukrittayakamee $\mathrm{S}$, Gruner $\mathrm{AC}$, Renia L, Letourneur $\mathrm{F}$, Looareesuwan S, White NJ, Snounou G: Practical PCR genotyping protocols for Plasmodium vivax using Pvcs and Pvmsp1. Malar J 2005, 4:20.

14. Brito CF, Ferreira MU: Molecular markers and genetic diversity of Plasmodium vivax. Mem Inst Oswaldo Cruz 2011, 106(Suppl 1):12-26.

15. Russell B, Suwanarusk R, Lek-Uthai U: Plasmodium vivax genetic diversity: microsatellite length matters. Trends Parasitol 2006, 22:399-401.

16. Anderson TJ, Su XZ, Bockarie M, Lagog M, Day KP: Twelve microsatellite markers for characterization of Plasmodium falciparum from finger-prick blood samples. Parasitology 1999, 119(Pt 2):113-125.

17. Imwong M, Nair S, Pukrittayakamee S, Sudimack D, Williams JT, Mayxay M, Newton PN, Kim JR, Nandy A, Osorio L, Carlton JM, White NJ, Day NP, Anderson TJ: Contrasting genetic structure in Plasmodium vivax populations from Asia and South America. Int J Parasitol 2007. 37:1013-1022

18. Koepfli C, Mueller I, Marfurt J, Goroti M, Sie A, Oa O, Genton B, Beck HP, Felger I: Evaluation of Plasmodium vivax genotyping markers for molecular monitoring in clinical trials. J Infect Dis 2009, 199:1074-1080.

19. Karunaweera ND, Ferreira MU, Munasinghe A, Barnwell JW, Collins WE, King CL, Kawamoto F, Hartl DL, Wirth DF: Extensive microsatellite diversity in the human malaria parasite Plasmodium vivax. Gene 2008, 410:105-112.

20. Gunawardena S, Karunaweera ND, Ferreira MU, Phone-Kyaw M, Pollack RJ, Alifrangis M, Rajakaruna RS, Konradsen F, Amerasinghe PH, Schousboe ML, Galappaththy GN, Abeyasinghe RR, Hartl DL, Wirth DF: Geographic structure of Plasmodium vivax: microsatellite analysis of parasite populations from Sri Lanka, Myanmar, and Ethiopia. Am J Trop Med Hyg 2010, 82:235-242.

21. Ellegren $\mathrm{H}$ : Microsatellites: simple sequences with complex evolution. Nat Rev Genet 2004, 5:435-445

22. Imwong M, Sudimack D, Pukrittayakamee S, Osorio L, Carlton JM, Day NP, White NJ, Anderson TJ: Microsatellite variation, repeat array length, and population history of Plasmodium vivax. Mol Biol Evol 2006, 23:1016-1018.

23. Ranjitkar S, Schousboe ML, Thomsen TT, Adhikari M, Kapel CM, Bygbjerg IC, Alifrangis M: Prevalence of molecular markers of anti-malarial drug resistance in Plasmodium vivax and Plasmodium falciparum in two districts of Nepal. Malar J 2011, 10:75.

24. Leslie T, Mayan MI, Hasan MA, Safi MH, Klinkenberg E, Whitty CJ, Rowland M: Sulfadoxine-pyrimethamine, chlorproguanil-dapsone, or chloroquine for the treatment of Plasmodium vivax malaria in Afghanistan and Pakistan: a randomized controlled trial. JAMA 2007, 297:2201-2209.

25. Leslie T, Mayan I, Mohammed N, Erasmus P, Kolaczinski J, Whitty CJ, Rowland M: A randomised trial of an eight-week, once weekly primaquine regimen to prevent relapse of Plasmodium vivax in Northwest Frontier Province, Pakistan. PLoS One 2008, 3:e2861.

26. Ord RL, Tami A, Sutherland CJ: ama1 genes of sympatric Plasmodium vivax and P. falciparum from Venezuela differ significantly in genetic diversity and recombination frequency. PLoS One 2008, 3:e3366.

27. Wooden J, Kyes S, Sibley CH: PCR and strain identification in Plasmodium falciparum. Parasitol Today 1993, 9:303-305.

28. Pearce RJ, Drakeley C, Chandramohan D, Mosha F, Roper C: Molecular determination of point mutation haplotypes in the dihydrofolate reductase and dihydropteroate synthase of Plasmodium falciparum in three districts of Northern Tanzania. Antimicrob Agents Chemother 2003, 47:1347-1354 
29. Goudet J: FSTAT, A Program to Estimate and Test Gene Diversities and Fixation Indices. [2.9.3]. ; 2001. Ref Type: Computer Program, http://www.unil.ch/izea/ softwares/Fstat.html. Updated from Goudet (1995).

30. Arlekin: An Integrated Software for Population Genetics Data Analysis. [3.11]; 2012. Ref Type: Computer Program.

31. Google: Google Earth. 2014. 24-8-2014. Ref Type: Online Source.

32. Orjuela-Sanchez P, da Silva NS, da Silva-Nunes M, Ferreira MU: Recurrent parasitemias and population dynamics of Plasmodium vivax polymorphisms in rural Amazonia. Am J Trop Med Hyg 2009, 81:961-968.

33. Bruce MC, Galinski MR, Barnwell JW, Donnelly CA, Walmsley M, Alpers MP, Walliker D, Day KP: Genetic diversity and dynamics of Plasmodium falciparum and $P$. vivax populations in multiply infected children with asymptomatic malaria infections in Papua New Guinea. Parasitology 2000, 121(Pt 3):257-272.

34. Kolakovich KA, Ssengoba A, Wojcik K, Tsuboi T, Al-Yaman F, Alpers M, Adams JH: Plasmodium vivax: favored gene frequencies of the merozoite surface protein-1 and the multiplicity of infection in a malaria endemic region. Exp Parasitol 1996, 83:11-19.

35. Ferreira MU, Karunaweera ND, da Silva-Nunes M, da Silva NS, Wirth DF, Hartl DL: Population structure and transmission dynamics of Plasmodium vivax in rural Amazonia. J Infect Dis 2007, 195:1218-1226.

36. Cui L, Escalante AA, Imwong M, Snounou G: The genetic diversity of Plasmodium vivax populations. Trends Parasitol 2003, 19:220-226.

doi:10.1186/1475-2875-13-392

Cite this article as: Schousboe et al:: Global and local genetic diversity at two microsatellite loci in Plasmodium vivax parasites from Asia, Africa and South America. Malaria Journal 2014 13:392.

\section{Submit your next manuscript to BioMed Central and take full advantage of:}

- Convenient online submission

- Thorough peer review

- No space constraints or color figure charges

- Immediate publication on acceptance

- Inclusion in PubMed, CAS, Scopus and Google Scholar

- Research which is freely available for redistribution 